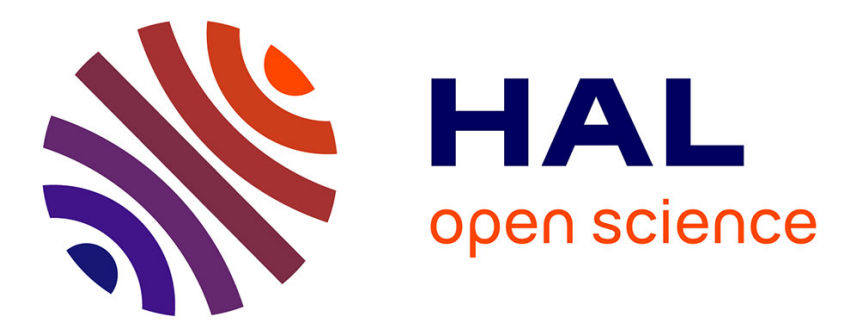

\title{
Component Design and Adaptation Based on Behavioral Contracts
}

\author{
Samir Chouali, Sebti Mouelhi, Hassan Mountassir
}

\section{To cite this version:}

Samir Chouali, Sebti Mouelhi, Hassan Mountassir. Component Design and Adaptation Based on Behavioral Contracts. International Workshop: Formal models for mastering multifaceted systems, Oct 2018, Marrakesh, Morocco. pp.217 - 230. hal-01992707

\section{HAL Id: hal-01992707 https://hal.science/hal-01992707}

Submitted on 24 Jan 2019

HAL is a multi-disciplinary open access archive for the deposit and dissemination of scientific research documents, whether they are published or not. The documents may come from teaching and research institutions in France or abroad, or from public or private research centers.
L'archive ouverte pluridisciplinaire HAL, est destinée au dépôt et à la diffusion de documents scientifiques de niveau recherche, publiés ou non, émanant des établissements d'enseignement et de recherche français ou étrangers, des laboratoires publics ou privés. 


\title{
Component Design and Adaptation Based on Behavioral Contracts
}

\author{
Samir Chouali ${ }^{1}$, Sebti Mouelhi ${ }^{2}$, and Hassan Mountassir ${ }^{1}$ \\ 1 Univ. Bourgogne Franche-Comté, FEMTO-ST Institute/CNRS, Besançon, France \\ schouali@femto-st.fr, hmountas@femto-st.fr \\ 2 ECE Paris - Graduate School of Engineering, Paris, France \\ sebti.mouelhi@ece.fr
}

\begin{abstract}
In this paper, our objective is to propose an adaptation approach to generate a component adaptor that ensures a correct interaction between mismatched components. Compared to the related works on component adaptation, the originality of our proposition relies on two main contributions. In the first, we design component behavioral contracts in order to generate component adaptor. So, we propose to specify component interfaces as behavioral contracts, to enrich the exhibited informations in component interfaces. Our behavioral contracts express all component facets: their action signatures, their actions semantics, and their protocol. We consider that these informations are important when generating component adaptors. In the second contribution, we propose to specify component behavioral contracts with the formalism based on interface automata that we enrich to specify the semantics of component actions. So, our adaptation approach is also an extension of the interface automata approach to handle the problem of component adaptation.
\end{abstract}

Keywords: Components · behavioral contracts · adaptation.

\section{Introduction}

The development of component-based systems is principally based on component reusability which allows the use of components in diverse environments without affecting their codes. However, in many cases, reusability is constrained with mismatches that may occur between components and their new environments during their interaction. The mismatches are caused by components that do not match perfectly the requirements of their environment. In this case, component adaptation should be performed in order to generate software entities, called adaptors, capable of enabling a correct interaction between components when mismatches occur.

In this paper, we focus on adapting components whose interfaces are described with behavioral contracts, which exhibit all component facets at the levels of action signatures (signatures of component operations), component protocols (scheduling of operation calls), and action semantics (semantic of component operations). We believe that consideration of all these informations in component interfaces lead to generate suitable and reliable adaptors. To specify formally component contracts, we propose to exploit the interface automata 


\section{S. Chouali et al.}

formalism [1] that we enrich by the semantic of component actions, because interface automata express only the scheduling of components actions without their semantics. So, we annotate the actions in interface automata by pre and post-conditions expressed on their parameters. This new formalism led us to adapt the compatibility verification approach, based on interface automata, to handle with the semantic of actions, because the adaptor generation relies on the verification of component compatibility.

Previously, we treated only adaptation at the protocol level [6]. Our purpose was to generate automatically an adaptor (interface automaton in-the-middle) for exactly two component interface automata according to a mapping that establishes a number of rules relating their mismatched input and output actions. In this paper, the main contribution relies on proposing a methodological approach to treat the problem of component adaptation at signature, semantic, and protocol levels, by exploiting component behavioral contracts. We show how to cooperate between the adaptation at the protocol level, and the semantic adaptation to generate a suitable adaptor for components specified with enriched interface automata that specify component contracts.

The paper is organized as follows. In Section 2, we present the formalization of component behavioral contracts with the interface automata, enriched with the semantics of component actions. In Section 3, we show how to verify the compatibility between components specified with behavioral contracts. When the compatibility does not hold between components, we present in Section 4 the specification of the mapping rules between the mismatched components that we exploit to generate adaptors. Section 5 , is dedicated to present our proposition to adapt components at signature, semantic, and protocol levels. Finally, we discuss the related work to our approach in Section 6 and conclude the paper in Section 7.

\section{Component Behavioral Contracts}

Interface automata (IAs) have been defined by L. Alfaro and T. Henzinger [1], to model the behavior of software component. Every component is described with an interface, which is specified with one interface automaton. This latter describes the scheduling of input, output, and hidden component actions, such that, input actions are used to model methods that can be called, and the end of receiving messages from communication channels, as well as the return values from such calls. Output actions are used to model method calls, message transmissions via communication channels, and exceptions that occur during the methods executions. Local operations are called hidden actions. The alphabet of an interface automaton consists of the names of actions annotated by "?" for input actions, by "!" for output actions, and by ";" for hidden actions. In the interface automata approach, the verification of the compatibility between two component is based on the composition of their interface automata, which is achieved by synchronizing their shared input and output actions. The compatibility holds between two interface automata where there is an environment (third component) which prevents the reachability of illegal states (states 
where the synchronization between the shared actions is not achieved) in their composition. This approach is considered optimistic because the existence of illegal states in the composition is not sufficient to decide on the incompatibility between components. The composition approach of the other automata-based formalisms describing the interface protocols of components are considered pessimistic.

In this paper, we propose to specify component behavioral contracts with interface automata formalism, enriched with the explicit description of the semantics of each action. These contracts specify component behaviors by showing the scheduling of the actions calls, and the interface automata formalism is suitable to specify component behaviors. However our behavioral contracts should express also the semantics of component actions, with pre and post conditions that should be satisfied by the environment in order to call or to provide component actions. And interface automata are not enough expressive to specify the semantics of component actions, therefore we propose to enrich this formalism to cope with action semantics.

In our proposal, we consider that the signature of an input (resp. output) component action $a$ is of the form $a\left(i_{1}, \ldots, i_{n}\right) \rightarrow(o)$. The set $P_{a}^{\mathrm{i}}=\left\{i_{1}, \ldots, i_{n}\right\}$ represents the set of input parameters of $a$. The set of output parameters $P_{a}^{\circ}$ is defined by the singleton $\{o\}$ (we assume that an action has at most a unique return value). The set of all parameters of an action $a$ is denoted by $P_{a}$. The absence of input or output parameters is denoted by (). For a parameter $p$, we define a domain $D_{p}$ which is a set of values that $p$ can take. The semantics of actions is represented by the pre and post-conditions defined on action parameters. We express these conditions as formulas of the first-order logic. Given a set of variables $V$, we denote by $\operatorname{Preds}(V)$, the set of first-order logic predicates whose free variables belong to $V$.

Definition 1 (Interface Automaton for a behavioral contract) Let $B$ be a behavioral contract associated to a component interface. An interface automaton to specify $B$ is a tuple $A=\left\langle\mathrm{S}_{A}, \mathrm{i}_{A}, \Sigma_{A}^{\mathrm{I}}, \Sigma_{A}^{\mathrm{O}}, \Sigma_{A}^{\mathrm{H}}, \delta_{A}, \Psi_{A}\right\rangle$ such that:

$-S_{A}$ is a finite set of states. A is called empty iff $S_{A}=\emptyset$;

$-i_{A} \in S_{A}$ is the initial state;

$-\Sigma_{A}^{\mathrm{I}}, \Sigma_{A}^{\mathrm{O}}$, and $\Sigma_{A}^{\mathrm{H}}$ are respectively the sets of names of input, output, and

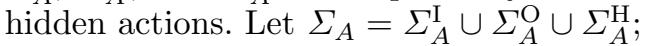

$-\delta_{A} \subseteq S_{A} \times \Sigma_{A} \times S_{A}$ is the set of transitions betweens states;

- $\Psi_{A}$ is a function, $\Psi_{A}: \Sigma_{A} \mapsto \operatorname{Preds}\left(P_{a}^{\mathrm{i}}\right) \times \operatorname{Preds}\left(P_{a}^{\mathrm{i}} \cup P_{a}^{\mathrm{o}}\right)$, that associates, for each action $\mathrm{a} \in \Sigma_{A}$, a tuple $\left\langle\operatorname{Pre}_{\Psi_{A}(a)}\right.$, $\left.\operatorname{Post}_{\Psi_{A}(a)}\right\rangle$ that represents the pre and post conditions of component actions.

We require that interface automata are deterministic, i.e. for all $\left(s, a, s_{1}\right) \in$ $\delta_{A}$ and $\left(s, a, s_{2}\right) \in \delta_{A}$, we have $s_{1}=s_{2}$.

The set $\Sigma_{A}^{\text {ext }}$ of external actions of interface automaton $A$ is defined by the union $\Sigma_{A}^{\mathrm{I}} \cup \Sigma_{A}^{\mathrm{O}}$. The set $\Sigma_{A}^{\text {loc }}$ of locally controlled actions of $A$ is defined by the union $\Sigma_{A}^{\mathrm{O}} \cup \Sigma_{A}^{\mathrm{H}}$. We define by $\Sigma_{A}^{\mathrm{I}}(s), \Sigma_{A}^{\mathrm{O}}(s), \Sigma_{A}^{\mathrm{H}}(s), \Sigma_{A}^{\text {ext }}(s)$, and $\Sigma_{A}^{\text {loc }}(s)$ respectively the input, output, hidden, external and locally controlled actions enabled from $s$. The set $\Sigma_{A}(s)$ includes all the enabled actions from $s$. 
S. Chouali et al.

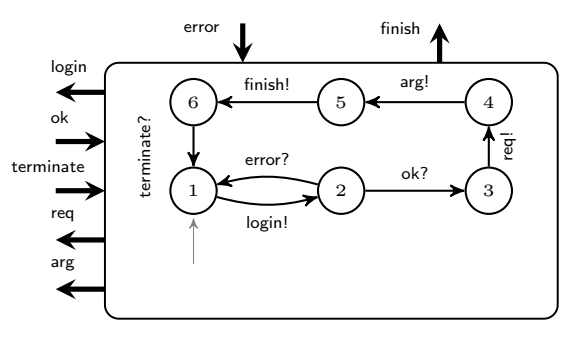

Client

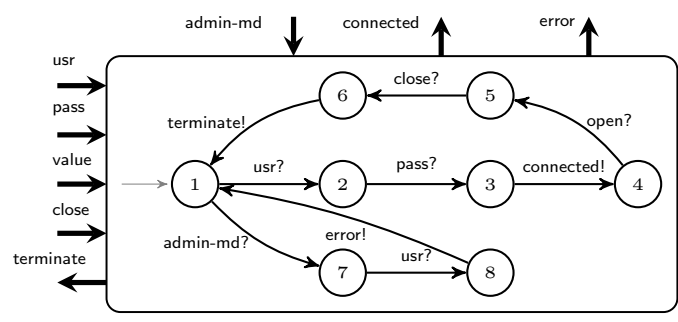

Server

Fig. 1. A variant of a client/server system

Example 1. Let us consider the two composable interface automata Client and Server, that specify component behavioral contracts, shown in Figure 1. After authentication, Client sends a request req! to open a file in read-only or write mode. After that, it sends an action arg! containing the name of a file to be open. Server receives the two actions by executing an action open? that open the file in readonly or write mode. After using the file, Client sends a signal finish! indicating to Server that the file is ready to be closed (action close?). Finally, Server sends a signal terminate! to terminate the session. The action admin-md? is a super signal received from the administrator of the system to open a super user session. When a client username is received by the server after receiving the admin-md! signal from an administrator, then an error is detected. For example, the signatures and the semantics of the action login in Client and usr in Server are defined as follows.

Signatures: login (uname, passwd,lu,lp) $\rightarrow$ (exist), usr (username, lengthu) $\rightarrow()$.

The semantics of the action login is defined as:

$\operatorname{Pre}_{\Psi_{\text {Client }}(\operatorname{login})} \equiv 1<\mathrm{lu} \leq 20 \wedge 8 \leq \mathrm{lp} \leq 10$,

Post $_{\Psi_{\text {Chient }}(\operatorname{login})} \equiv$ exist $=1 \vee$ exist $=0$

The semantics of the action usr is defined as:

$\operatorname{Pre}_{\Psi_{\text {Server }(\text { usr })}} \equiv 1<$ lengthu $\leq 30$, Post $_{\Psi_{\text {Server }}(\text { usr })} \equiv$ true

\section{Component Compatibility Based on Behavioral Contracts}

In this section we show how to verify the compatibility between two components specified with their behavioral contracts. Our proposition relies on the extension of the interface automata approach to cope with the semantics of component actions expressed with their pre and post condition on their parameters. To verify the compatibility between two components that are specified with two 
interface automata $A_{1}$ and $A_{2}$, we have first to verify their composability and then compute their composition by their synchronized product.

Before defining the composition between, $A_{1}$ and $A_{2}$, we present in the following the conditions that should be respected by both automata, that specify component behavioral contracts, in order to authorize their composition.

The Composability conditions: Two interface automata $A_{1}$ and $A_{2}$ associated to two behavioral contracts are composable if :

- The condition on the non shared input and output actions is satisfied:

$$
\Sigma_{A_{1}}^{\mathrm{I}} \cap \Sigma_{A_{2}}^{\mathrm{I}}=\Sigma_{A_{1}}^{\mathrm{O}} \cap \Sigma_{A_{2}}^{\mathrm{O}}=\Sigma_{A_{1}}^{\mathrm{H}} \cap \Sigma_{A_{2}}=\Sigma_{A_{2}}^{\mathrm{H}} \cap \Sigma_{A_{1}}=\emptyset .
$$

- The condition on the shared actions is satisfied:

Shared $\left(A_{1}, A_{2}\right)=\left(\Sigma_{A_{1}}^{\mathrm{I}} \cap \Sigma_{A_{2}}^{\mathrm{O}}\right) \cup\left(\Sigma_{A_{2}}^{\mathrm{I}} \cap \Sigma_{A_{1}}^{\mathrm{O}}\right)$ is the set of shared input and output actions of $A_{1}$ and $A_{2}$. For each action $a \in \operatorname{Shared}\left(A_{1}, A_{2}\right)$ such that its signature is given by $a\left(i_{1}, \ldots, i_{n}\right) \rightarrow(o)$ in $A_{1}$ and by $a\left(i_{1}^{\prime}, \ldots, i_{n}^{\prime}\right) \rightarrow$ $\left(o^{\prime}\right)$ in $A_{2}$ then, $D_{i_{k}} \subseteq D_{i_{k}^{\prime}}$ for $1 \leq k \leq n$ and $D_{o} \subseteq D_{o^{\prime}}$ in the case where $a\left(i_{1}, \ldots, i_{n}\right) \rightarrow(o) \in \Sigma_{A_{1}}^{\mathrm{O}}$ and $a\left(i_{1}^{\prime}, \ldots, i_{n}^{\prime}\right) \rightarrow\left(o^{\prime}\right) \in \Sigma_{A_{2}}^{\mathrm{I}}$. Otherwise, $D_{i_{k}} \supseteq D_{i_{k_{f}}}$ for $1 \leq k \leq n$ and $D_{o} \supseteq D_{o^{\prime}}$. This property is called the domain inclusion of the parameters of shared actions. The intuition behind this condition comes from the fact that the output actions specify the method calls and the input ones specify the methods that can be called.

If the above conditions are satisfied between two interface automata $A_{1}$ and $A_{2}$, then we have to perform the renaming of parameter names in their pre and post-conditions in order to realize their composition.

Definition 2 (Parameter renaming) Given an action a in $\operatorname{Shared}\left(A_{1}, A_{2}\right)$, the signature of a is defined by a $\left(i_{1}, \ldots, i_{n}\right) \rightarrow(o)$ in $A_{1}$ and by a $\left(i_{1}^{\prime}, \ldots, i_{n}^{\prime}\right) \rightarrow\left(o^{\prime}\right)$ in $A_{2}$. The renaming of parameters in the semantics $\Psi_{A_{1}}(a)$ and $\Psi_{A_{2}}(a)$ is the substitution of $i_{1}^{\prime}$ by $i_{1}, \ldots, i_{n}^{\prime}$ by $i_{n}$, and $o^{\prime}$ by $o$ in $\operatorname{Pre}_{\Psi_{A_{2}}(a)}$ and $\operatorname{Post}_{\Psi_{A_{2}}(a)}$ or the opposite in $\operatorname{Pre}_{\Psi_{A_{1}}(a)}$ and $\operatorname{Post}_{\Psi_{A_{1}}(a)}$.

We denote by $\Psi_{A_{1} / A_{2}}(a)$ and $\Psi_{A_{2} / A_{1}}(a)$, the semantics of $a$ after the parameter renaming respectively in $A_{1}$ and $A_{2}$. We can now define properly the notion of the semantic compatibility of shared external actions.

Definition 3 (Semantic compatibility) Given an action a $\in \operatorname{Shared}\left(A_{1}, A_{2}\right)$, if one of the following conditions is true, then the action a in $A_{1}$ is semantically compatible with the same action a in $A_{2}$ i.e. $\operatorname{SemComp}_{a}\left(A_{1}, A_{2}\right)$ is true (otherwise $\neg \operatorname{SemComp}_{a}\left(A_{1}, A_{2}\right)$ is true ):

- if a $\in \Sigma_{A_{1}}^{\mathrm{O}} \wedge \operatorname{Pre}_{\Psi_{A_{1} / A_{2}}(a)} \Rightarrow \operatorname{Pre}_{\Psi_{A_{2} / A_{1}}(a)} \wedge \operatorname{Post}_{\Psi_{A_{2} / A_{1}}(a)} \Rightarrow \operatorname{Post}_{\Psi_{A_{1} / A_{2}}(a)}$,

- if a $\in \Sigma_{A_{1}}^{\mathrm{I}} \wedge \operatorname{Pre}_{\Psi_{A_{2} / A_{1}}(a)} \Rightarrow \operatorname{Pre}_{\Psi_{A_{1} / A_{2}}(a)} \wedge \operatorname{Post}_{\Psi_{A_{1} / A_{2}}(a)} \Rightarrow \operatorname{Post}_{\Psi_{A_{2} / A_{1}}(a)}$.

Definition 4 (Synchronized product $\otimes$ ) Given two composable interface automata $A_{1}$ and $A_{2}$, the synchronized product $A_{1} \otimes A_{2}$ of $A_{1}$ and $A_{2}$ is defined by: 


\section{S. Chouali et al.}

$$
\begin{aligned}
& -S_{A_{1} \otimes A_{2}}=S_{A_{1}} \times S_{A_{2}} \text { and } i_{A_{1} \otimes A_{2}}=\left(i_{A_{1}}, i_{A_{2}}\right) ; \Sigma_{A_{1} \otimes A_{2}}^{\mathrm{I}}=\left(\Sigma_{A_{1}}^{\mathrm{I}} \cup \Sigma_{A_{2}}^{\mathrm{I}}\right) \backslash \\
& \text { Shared }\left(A_{1}, A_{2}\right) \text {; } \\
& \text { - } \Sigma_{A_{1} \otimes A_{2}}^{\mathrm{O}}=\left(\Sigma_{A_{1}}^{\mathrm{O}} \cup \Sigma_{A_{2}}^{\mathrm{O}}\right) \backslash \operatorname{Shared}\left(A_{1}, A_{2}\right) ; \Sigma_{A_{1} \otimes A_{2}}^{\mathrm{H}}=\Sigma_{A_{1}}^{\mathrm{H}} \cup \Sigma_{A_{2}}^{\mathrm{H}} \cup\{a \in \\
& \text { Shared } \left.\left(A_{1}, A_{2}\right) \mid \operatorname{SemComp}\left(A_{1}, A_{2}\right)\right\} \text {; } \\
& \text { - }\left(\left(s_{1}, s_{2}\right), a,\left(s_{1}^{\prime}, s_{2}^{\prime}\right)\right) \in \delta_{A_{1} \otimes A_{2}} \text { iff } \\
& \text { - } a \notin \operatorname{Shared}\left(A_{1}, A_{2}\right) \wedge\left(s_{1}, a, s_{1}^{\prime}\right) \in \delta_{A_{1}} \wedge s_{2}=s_{2}^{\prime} \text { or } a \notin \operatorname{Shared}\left(A_{1}, A_{2}\right) \wedge \\
& \left(s_{2}, a, s_{2}^{\prime}\right) \in \delta_{A_{2}} \wedge s_{1}=s_{1}^{\prime} \text { or } a \in \operatorname{Shared}\left(A_{1}, A_{2}\right) \wedge\left(s_{1}, a, s_{1}^{\prime}\right) \in \delta_{A_{1}} \wedge \\
& \left(s_{2}, a, s_{2}^{\prime}\right) \in \delta_{A_{2}} \wedge \operatorname{SemComp}_{a}\left(A_{1}, A_{2}\right) \text {; } \\
& -\Psi_{A_{1} \otimes A_{1}} \text { is defined by: } \\
& \text { - } \Psi_{A_{1}} \text { for } a \in \Sigma_{A_{2}} \backslash \operatorname{Shared}\left(A_{1}, A_{2}\right) \text {; } \\
& \text { - } \Psi_{A_{2}} \text { for } a \in \Sigma_{A_{2}} \backslash \operatorname{Shared}\left(A_{1}, A_{2}\right) \text {; } \\
& \text { - }\left\langle\operatorname{Pre}_{\Psi_{A_{1}}(a)}, \text { Post }_{\Psi_{A_{2}}(a)}\right\rangle \text { for } a \in \operatorname{Shared}\left(A_{1}, A_{2}\right) \cap \Sigma_{A_{1}}^{\mathrm{O}} \text { such that } \operatorname{SemComp}_{a}\left(A_{1}, A_{2}\right) \text {; } \\
& \text { - }\left\langle\operatorname{Pre}_{\Psi_{A_{2}}(a)}, \text { Post }_{\Psi_{A_{1}}(a)}\right\rangle \text { for } a \in \operatorname{Shared}\left(A_{1}, A_{2}\right) \cap \Sigma_{A_{1}}^{\mathrm{I}} \text { such that } \operatorname{SemComp}_{a}\left(A_{1}, A_{2}\right) \text {; }
\end{aligned}
$$

The incompatibility between two interface automata $A_{1}$ and $A_{2}$ could happen due to (i) the existence of states $\left(s_{1}, s_{2}\right)$ in the product $A_{1} \otimes A_{2}$ such that there exists at least one action $a$ in $\operatorname{Shared}\left(A_{1}, A_{2}\right)$ enabled from $s_{1}$ and it is not from $s_{2}$ or inversely, or (ii) the action $a$ is enabled from $s_{1}$ and $s_{2}$ but $\neg \operatorname{SemComp}_{a}\left(A_{1}, A_{2}\right)$ is valid. These states are therefore illegal in the product $A_{1} \otimes A_{2}$.

Definition 5 (Illegal states) The set of illegal states, denoted by $\operatorname{Illegal}\left(A_{1}, A_{2}\right)$ $\subseteq S_{A_{1}} \times S_{A_{2}}$, in $A_{1} \otimes A_{2}$ is defined by $\left\{\left(s_{1}, s_{2}\right) \in S_{A_{1}} \times S_{A_{2}} \mid\left(\exists a \in \operatorname{Shared}\left(A_{1}, A_{2}\right) \mid\right.\right.$ the condition $C_{1} \oplus C_{2}$ is true $\left.\left.{ }^{3}\right)\right\}$.

$$
\begin{gathered}
C_{1}=\left(\begin{array}{c}
\left(a \in \Sigma_{A_{1}}^{\mathrm{O}}\left(s_{1}\right) \wedge a \notin \Sigma_{A_{2}}^{\mathrm{I}}\left(s_{2}\right)\right) \vee\left(a \in \Sigma_{A_{1}}^{\mathrm{O}}\left(s_{1}\right) \wedge a \in \Sigma_{A_{2}}^{\mathrm{I}}\left(s_{2}\right)\right. \\
\left.\wedge \neg \operatorname{Sem} \operatorname{Comp}_{a}\left(A_{1}, A_{2}\right)\right)
\end{array}\right) \\
C_{2}=\left(\begin{array}{c}
\left(a \in \Sigma_{A_{2}}^{\mathrm{O}}\left(s_{2}\right) \wedge a \notin \Sigma_{A_{1}}^{\mathrm{I}}\left(s_{1}\right)\right) \vee\left(a \in \Sigma_{A_{2}}^{\mathrm{O}}\left(s_{2}\right) \wedge a \in \Sigma_{A_{1}}^{\mathrm{I}}\left(s_{1}\right)\right. \\
\left.\wedge \neg \operatorname{Sem} \operatorname{Comp}_{a}\left(A_{1}, A_{2}\right)\right)
\end{array}\right)
\end{gathered}
$$

Reaching states in $\operatorname{Illegal}\left(A_{1}, A_{2}\right)$ is not sufficient to decide that $A_{1}$ and $A_{2}$ are incompatible (according to optimistic approach). Indeed, in this approach, if there is at least one environment that requests the appropriate input actions in $A_{1} \otimes A_{2}$, and allows the no reachability of illegal states, then $A_{1}$ and $A_{2}$ can be assembled without producing deadlocks. The composition of $A_{1}$ and $A_{2}$, denoted by $A_{1} \| A_{2}$, is the restriction of their product to the set of states called compatible, denoted by $\operatorname{Comp}\left(A_{1}, A_{2}\right)$. They are the states through which the interaction between the two components of $A_{1}$ and $A_{2}$ passes without having the risk of reaching illegal states by enabling only the locally controllable actions (input and hidden actions). The verification steps in this approach are similar to those described in [1], except that we consider the semantics of actions during the compatibility check by verifying the condition of semantic compatibility between the shared actions.

\footnotetext{
${ }^{3} \oplus$ is $\mathrm{XOR}$
} 
Component Design and Adaptation Based on Behavioral Contracts

\section{Component Behavioral Contracts and Mismatches}

The definitions of component interface mismatches $[5,2,4]$ are essentially due to the reuse of components in a system design which is often harmed by mismatch cases such as: (i) names of exchanged messages between components do not correspond which may lead to deadlock situations, components regularly interact on the same action names; (ii) the orderings of messages or actions in both component protocols do not correspond; (iii) an action in a component that has no counterpart in the other one, or correspond to more than one action.

For component behavioral contracts specified with interface automata, the behavioral mismatch cannot be detected by applying the synchronized product between two composable interface automata as it was defined in Definition 4, because the case where there is no correspondence between the action names leads to them being absent from the set of shared actions. Thus, all of mismatched actions are interleaved asynchronously in the product. To avoid this constraint, our adaptation specification starts by establishing an abstract way to denote the composition requirements. We corroborate the explicit description of interactions between components thanks to rules. They relate the mismatched actions used in different components which are supposed to implement some interactions. Rules relate actions even if they do not really label some transitions in the automaton as required by the optimistic approach of interface automata.

The minimal adaptor specification of two interface automata $A_{1}$ and $A_{2}$ is the set of rules called a mapping. The mapping does not represent any behavioural detail about the adaptor.

Definition 6 (Rules and Mappings) A rule $\alpha$ for two composable interface automata $A_{1}$ and $A_{2}$, is a pair $\left\langle L_{1}, L_{2}\right\rangle \in\left(2^{\Sigma_{A_{1}}^{\mathrm{O}}} \times 2^{\Sigma_{A_{2}}^{\mathrm{I}}}\right) \cup\left(2^{\Sigma_{A_{1}}^{\mathrm{I}}} \times 2^{\Sigma_{A_{2}}^{\mathrm{O}}}\right)^{4}$ such that $\left(L_{1} \cup L_{2}\right) \cap \operatorname{Shared}\left(A_{1}, A_{2}\right)=\emptyset$ and if $\left|L_{1}\right|>1\left(\right.$ or $\left.\left|L_{2}\right|>1\right)$ then $\left|L_{2}\right|=1$ (or $\left.\left|L_{1}\right|=1\right)$;

$A$ mapping $\Phi\left(A_{1}, A_{2}\right)$ for two composable interface automata $A_{1}$ and $A_{2}$ is a set of rules $\alpha_{i}$, for $1 \leq i \leq\left|\Phi\left(A_{1}, A_{2}\right)\right|$.

According to the above definition, a rule in our approach deals with oneto-one, many-to-one, and one-to-many correspondences between actions. More clearly, the adaptation may in general relate either an action or a group of actions of one automaton with one action in the other. For instance, a client authenticates itself by sending first its user name and then a password while the server accepts both data in a single login shot. We denote the set of the mismatched actions by $\operatorname{Mismatch}_{\Phi}\left(A_{1}, A_{2}\right)=\left\{a \in \Sigma_{A_{1}}^{e x t} \cup \Sigma_{A_{2}}^{e x t} \mid \exists \alpha \in \Phi\left(A_{1}, A_{2}\right)\right.$ . $\left.a \in \Pi_{1}(\alpha) \vee \mathrm{a} \in \Pi_{2}(\alpha)\right\}^{5}$.

Example 2. To illustrate the mapping relation, we define this latter between the actions of the interface automata Client and server as described in Figure 1 by: $\Phi($ Client, Server $)=\{\langle\{$ login $\},\{$ usr, pass $\}\rangle,\langle\{$ finish $\},\{$ close $\}\rangle,\langle\{$ ok $\},\{$ connected $\}\rangle$ $\langle\{$ req,arg $\},\{$ open $\}\rangle\}$. The set $\operatorname{Shared}\left(A_{1}, A_{2}\right)=\{$ error,terminate $\}$.

\footnotetext{
${ }^{4}$ For some set $S, 2^{S}$ is its power set.

${ }^{5} \Pi_{1}(\langle a, b\rangle)=a$ and $\Pi_{2}(\langle a, b\rangle)=b$ are respectively the projection on the first element and the second element of the couple $\langle a, b\rangle$.
} 


\section{S. Chouali et al.}

Given two composable interface automata $A_{1}$ and $A_{2}$ and a mapping $\Phi\left(A_{1}\right.$, $\left.A_{2}\right)$, if $\Phi\left(A_{1}, A_{2}\right)=\emptyset$, the adaptation of $A_{1}$ and $A_{2}$ has no sense and their synchronization is defined by their product $A_{1} \otimes A_{2}$ as it was defined in section 3 . Otherwise, we proceed on two steps: (i) we check first the semantic adaptability between the mismatched actions in the mapping $\Phi\left(A_{1}, A_{2}\right)$. (ii)if the semantic adaptability check was successfully made without giving rise to incompatibilities, we generate the adaptor of $A_{1}$ and $A_{2}$ according to the mapping $\Phi\left(A_{1}, A_{2}\right)$. If the generated adaptor is non-empty and it is compatible with both of $A_{1}$ and $A_{2}$, we say that $A_{1}$ and $A_{2}$ are adaptable.

\section{Component Adaptation}

In this section we present our approach to adapt components specified with behavioral contracts.

\subsection{Semantic Adaptability}

The semantic adaptability between the mismatched actions of two composable interface automata has to be made before generating the adaptor. The mismatched actions have to respect some constraints at the level of their semantics. Let us consider two interface automata $A_{1}$ and $A_{2}$ and a given mapping $\Phi\left(A_{1}, A_{2}\right)$. To perform the semantic adaptability check between $A_{1}$ and $A_{2}$ according to $\Phi\left(A_{1}, A_{2}\right)$, it is required that for each rule $\alpha=\left\langle L_{1}, L_{2}\right\rangle \in \Phi\left(A_{1}, A_{2}\right)$ the following conditions hold:

1. $\sum_{a \in L_{1}}\left|P_{a}^{\mathrm{i}}\right|=\sum_{b \in L_{2}}\left|P_{b}^{\mathrm{i}}\right|$

2. $\sum_{a \in L_{1}}\left|P_{a}^{\mathrm{o}}\right|=\sum_{b \in L_{2}}\left|P_{b}^{\mathrm{o}}\right|$;

3. if $\left|L_{1}\right|=1$ and $\left|L_{2}\right| \geq 1$ where $L_{1}=\{a\}, L_{2}=\left\{b_{1}, \ldots, b_{\left|L_{2}\right|}\right\}$, and $P_{a}^{o}=\left\{o_{a}\right\}$ then there exists exactly one action $b_{k} \in L_{2}\left(1 \leq k \leq\left|L_{2}\right|\right)$ such that $P_{b_{k}}^{\mathrm{o}}=$ $\left\{o_{b_{k}}\right\}, P_{b_{l}}^{\circ}=\emptyset$ for $1 \leq l \leq\left|L_{2}\right|$ and $l \neq k$, and the two output parameters $o_{a}$ and $o_{b_{k}}$ have to satisfy the domain inclusion condition:

- if $L_{1} \subseteq \Sigma_{A_{1}}^{\mathrm{O}}$, then $D_{o_{a}} \subseteq D_{o_{b_{k}}}$;

- else $D_{o_{a}} \supseteq D_{o_{b_{k}}}$;

$\theta_{\alpha}$ denotes the tuple $\left(a, b_{k}\right)$. If $P_{a}^{\mathrm{o}}=\{\},\left(a, b_{k}\right)$ is not defined;

4. the condition is analogous to the previous one with $\left|L_{1}\right| \geq 1$ and $\left|L_{2}\right|=1$ where $L_{1}=\left\{a_{1}, \ldots, a_{\left|L_{1}\right|}\right\}$ and $L_{2}=\{b\}$;

5. there exists a function $\varphi_{\alpha}^{\mathrm{i}}: \bigcup_{a \in L_{1}} P_{a}^{\mathrm{i}} \rightarrow \bigcup_{b \in L_{2}} P_{b}^{\mathrm{i}}$ that associates each input parameter $p$ of actions in $L_{1}$ with an input parameter $q$ of actions in $L_{2}$. The function $\varphi_{\alpha}^{\mathrm{i}}$ have to satisfy the domain inclusion condition:

- if $L_{1} \subseteq \Sigma_{A_{1}}^{\mathrm{O}}$, then $D_{p} \subseteq D_{\varphi_{\alpha}^{\mathrm{i}}(p)}$ where $p \in \bigcup_{a \in L_{1}} P_{a}^{\mathrm{i}}$;

- else $D_{\varphi_{\alpha}^{\mathrm{i}}(p)} \subseteq D_{p}$ where $p \in \bigcup_{a \in L_{1}} P_{a}^{\mathrm{i}}$.

The first and the second conditions state that the number of input (respectively output) parameters of actions in $L_{1}$ is equal to the number of input (respectively output) parameters of actions in $L_{2}$. The third condition states the relations between the output parameter of the action $a \in L_{1}$ and the one of the 
action $b_{k} \in L_{2}$. We assume that the other actions in $L_{2} \backslash\left\{b_{k}\right\}$ have no output parameters. The intuition behind these conditions is to avoid conflicts between the pre and post-conditions during the semantic adaptability check by ensuring the equality between the number of input and output parameters.

The renaming of the input and output parameter in the semantics of actions in $\operatorname{Mismatch}_{\Phi}\left(A_{1}, A_{2}\right)$ is defined as follows. For all $a \in L_{1}$ and $b \in L_{2}$, the parameter renaming is defined by the substitution of each input parameter $i$ of $a$ in $\operatorname{Pre}_{\Psi_{A_{1}}(a)}$ and $\operatorname{Post}_{\Psi_{A_{1}}(a)}$ by $\varphi_{\alpha}^{\mathrm{i}}(i)$ or the substitution of each input parameter $i^{\prime}$ of $b$ in $\operatorname{Pre}_{\Psi_{A_{2}}(b)}$ and $\operatorname{Post}_{\Psi_{A_{2}}(b)}$ by $\varphi_{\alpha}^{\mathrm{i}}{ }^{-1}\left(i^{\prime}\right)^{6}$. If the couple $\theta_{\alpha}=(a, b)$ exists, the parameter renaming is defined by the substitution of the output parameter $o_{a}$ in Post $_{\Psi_{A_{1}}(a)}$ by $o_{b}$ or the substitution of the output parameter $o_{b}$ in Post $_{\Psi_{A_{2}}(b)}$ by $o_{a}$.

We denote by $\Psi_{A_{1} / \alpha}(a)$ and $\Psi_{A_{2} / \alpha}(b)$ respectively the semantics of actions in $\Pi_{1}(\alpha)$ and actions in $\Pi_{2}(\alpha)$ after the parameter renaming.

Definition 7 (Semantic Adaptability) Given two composable interface automata $A_{1}$ and $A_{2}$ and an adaptation mapping $\Phi\left(A_{1}, A_{2}\right)$ such that the conditions $1,2,3,4$, and 5 introduced in Section 5.1 are satisfied, the semantic adaptability $\operatorname{SemAdap}_{\alpha}\left(A_{1}, A_{2}\right)$ of a rule $\alpha$ in $\Phi\left(A_{1}, A_{2}\right)$ is satisfied iff the following conditions are fulfilled:

1. If $\Pi_{1}(\alpha) \subseteq \Sigma_{A_{1}}^{\mathrm{O}}$, then

$$
\left.\begin{array}{rl}
\bigwedge_{a \in \Pi_{1}(\alpha)} \operatorname{Pre}_{\Psi_{A_{1} / \alpha}(a)} & \Rightarrow \bigwedge_{b \in \Pi_{2}(\alpha)} \operatorname{Pre}_{\Psi_{A_{2} / \alpha}(b)} \\
\bigwedge_{a \in \Pi_{1}(\alpha)} \operatorname{Post}_{\Psi_{A_{1} / \alpha}(a)} & \Leftarrow \bigwedge_{b \in \Pi_{2}(\alpha)} \operatorname{Post}_{\Psi_{A_{2} / \alpha}(b)}
\end{array}\right)
$$

2. If $\Pi_{1}(\alpha) \subseteq \Sigma_{A_{1}}^{\mathrm{I}}$, then the condition is analogous to the previous one by inversing the implications.

We say that $A_{1}$ and $A_{2}$ are semantically adaptable according to the mapping $\Phi\left(A_{1}, A_{2}\right)$ if the semantic adaptability of each rule $\alpha \in \Phi\left(A_{1}, A_{2}\right)$ holds.

The semantic adaptability conditions are stated in a similar way as the semantic compatibility of the shared actions defined in Definition 3 except that for adaptation, we treat sets of mismatched actions associated by the rules of the mapping.

Example 3. To illustrate mismatches between actions belonging to two behavioral contracts, we consider the two composable interface automata Client and Server, that specify component behavioral contracts, shown in Figure 1 and a mapping $\Phi($ Client,Server $)$ as defined in Example 2.

The mismatched actions are described and classified by the rules in Table 1. The function $\varphi_{\alpha_{2}}^{\mathrm{i}}$ is defined by $\{m s g \mapsto l o g m s g\}$. The function $\varphi_{\alpha_{4}}^{\mathrm{i}}$ is not

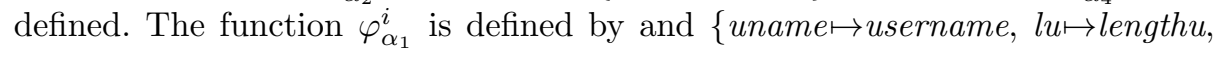


S. Chouali et al.

Table 1. The signatures of actions in Mismatch $_{\Phi}($ Client,Server $)$

\begin{tabular}{|l|l|l|}
\hline & Client & Server \\
\hline$\alpha_{1}$ & $\operatorname{login}($ uname,passwd,lu,lp $) \rightarrow($ exist $)$ & usr $($ username,lengthu $) \rightarrow($ ) \\
\cline { 3 - 3 } & & pass (password,lengthp $) \rightarrow($ exist $)$ \\
\hline$\alpha_{2}$ & ok $(\mathrm{msg}) \rightarrow()$ & connected $($ logmsg $) \rightarrow()$ \\
\hline$\alpha_{3}$ & req(read $) \rightarrow()$ & open $($ readonly,filename $) \rightarrow($ open $)$ \\
\cline { 2 - 3 } & $\arg ($ file $) \rightarrow($ status $)$ & \\
\hline$\alpha_{4}$ & finish ()$\rightarrow($ status $)$ & close ()$\rightarrow($ closed $)$ \\
\hline
\end{tabular}

Table 2. The semantics of actions in $\operatorname{Mismatch}_{\Phi}($ Client,Server $)$

\begin{tabular}{|c|c|}
\hline Client & Server \\
\hline \multirow[t]{2}{*}{$\begin{array}{l}\operatorname{Pre}_{\Psi_{\text {Client }}(\text { login })} \equiv 1<\mathrm{lu} \leq 20 \wedge 8 \leq \mathrm{lp} \leq 10 \\
\operatorname{Post}_{\Psi_{\text {Client }}(\text { login })} \equiv \text { exist }=1 \vee \text { exist }=0\end{array}$} & $\begin{array}{l}\operatorname{Pre}_{\Psi_{\text {Server }}(u s r)} \equiv 1<\text { lengthu } \leq 30 \\
\operatorname{Post}_{\Psi_{\text {Server }}(\text { usr })} \equiv \text { true }\end{array}$ \\
\hline & $\begin{array}{l}\operatorname{Pre}_{\Psi_{\text {Server }}(\text { pass })} \equiv 6 \leq \text { lengthp } \leq 10 \\
\operatorname{Post}_{\Psi_{\text {Server }}(\text { pass })} \equiv \text { exist }=1 \vee \text { exist }=0\end{array}$ \\
\hline $\begin{array}{l}\operatorname{Pre}_{\Psi_{\text {Client }}(o k)} \equiv \text { true } \\
\operatorname{Post}_{\Psi_{\text {Client }}(o k)} \equiv \text { true }\end{array}$ & $\begin{array}{l}\operatorname{Pre}_{\Psi_{\text {Server }}(\text { connected })} \equiv \text { true } \\
\text { Post }_{\Psi_{\text {Server }}(\text { connected })} \equiv \text { true }\end{array}$ \\
\hline $\begin{array}{l}\operatorname{Pre}_{\Psi_{\text {Client }}(r e q)} \equiv \text { read }=0 \vee \text { read }=1 \\
\operatorname{Post}_{\Psi_{\text {Client }}(\text { req })} \equiv \text { true }\end{array}$ & \multirow[t]{2}{*}{$\begin{array}{l}\operatorname{Pre}_{\Psi_{\text {Server }}(\text { open })} \equiv \text { readonly }=0 \vee \text { readonly }=1 \\
\operatorname{Post}_{\Psi_{\text {Server }}(\text { open })} \equiv \text { open }=0 \vee \text { open }=1\end{array}$} \\
\hline $\begin{array}{l}\operatorname{Pre}_{\Psi_{\text {Client }}(\text { arg })} \equiv \text { true } \\
\operatorname{Post}_{\Psi_{\text {Client }}(\text { arg })} \equiv \text { status }=0 \vee \text { status }=1\end{array}$ & \\
\hline $\begin{array}{l}\operatorname{Pre}_{\Psi_{\text {Client }}(\text { finish })} \equiv \text { true } \\
\operatorname{Post}_{\Psi_{\text {Client }}(\text { finish })} \equiv \text { status }=0 \vee \text { status }=1\end{array}$ & $\begin{array}{l}\operatorname{Pre}_{\Psi_{\text {Server }}(\text { close })} \equiv \text { true } \\
\operatorname{Post}_{\Psi_{\text {Server }}(\text { close })} \equiv \text { closed }=0 \vee \text { closed }=1\end{array}$ \\
\hline
\end{tabular}

passwd $\mapsto$ password, $l p \mapsto$ lengthp $\}$. The function $\varphi_{\alpha_{3}}^{\mathrm{i}}$ is defined by $\{$ read $\mapsto$ readonly, file $\mapsto$ filename $\}$. The function $\varphi_{\alpha_{4}}^{\mathrm{i}}$ is empty. $\theta_{\alpha_{1}}=$ (login,pass), $\theta_{\alpha_{2}}$ is not defined, $\theta_{\alpha_{3}}=($ arg,open $)$, and $\theta_{\alpha_{4}}=$ (finish,close). The parameters uname, passwd, username, password, msg, logmsg, file, and filename are strings. The parameters $l u$, lp, lengthu, lengthp, read, readonly, status, open, and closed are integers. As the reader can conclude, the conditions to perform the semantic adaptability check hold for all $\alpha$ in $\Phi\left(A_{1}, A_{2}\right)$ :

- for all $\alpha \in \Phi\left(A_{1}, A_{2}\right), \sum_{a \in \Pi_{1}(\alpha)}\left|P_{a}^{\mathrm{i}}\right|=\sum_{b \in \Pi_{2}(\alpha)}\left|P_{b}^{\mathrm{i}}\right|$ and $\sum_{a \in \Pi_{1}(\alpha)}\left|P_{a}^{\mathrm{o}}\right|=$ $\sum_{b \in \Pi_{2}(\alpha)}\left|P_{b}^{o}\right|$;

- the domain inclusion conditions are satisfied for $\theta_{*}$ and $\varphi_{*}^{i}$ where ${ }^{*} \in \Phi($ Client, Server).

The semantics of the mismatched actions respectively for Client and Server are listed in Table 2. After unifying the mismatched actions in Mismatch $_{\Phi}($ Client, Server), the reader can easily verify the semantic adaptability for all $\alpha$ in $\Phi($ Client, Server $)$ holds. For example, for the rule $\alpha_{1}$, 
Component Design and Adaptation Based on Behavioral Contracts

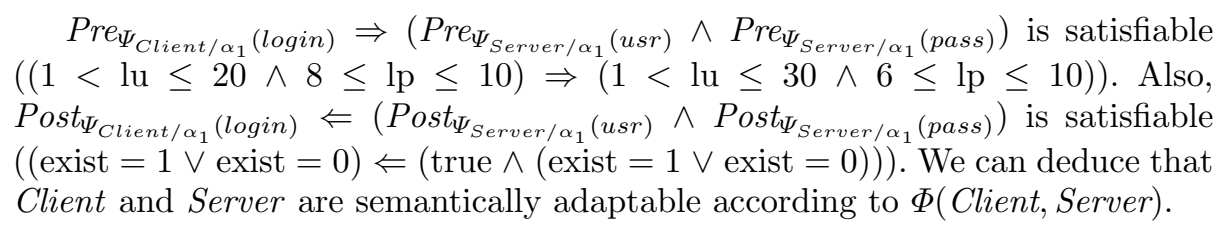

\subsection{Adaptor Specification and Construction}

After verifying the semantic adaptability between two composable interface automata $A_{1}$ and $A_{2}$ according to a mapping $\Phi\left(A_{1}, A_{2}\right)$, we treat in this section the interface automaton specification and construction of their adaptor. The adaptor must be composable with $A_{1}$ and $A_{2}$, and must also satisfy the mapping rules and respect the component protocols specified by $A_{1}$ and $A_{2}$.

Definition 8 (Adaptor) Given two composable interface automata $A_{1}, A_{2}$, and a mapping $\Phi\left(A_{1}, A_{2}\right)$, an adaptor for $A_{1}$ and $A_{2}$ according to the mapping $\Phi\left(A_{1}, A_{2}\right)$ is an interface automaton $A d=\left\langle S_{A d}, I_{A d}, \Sigma_{A d}^{\mathrm{I}}, \Sigma_{A d}^{\mathrm{O}}, \Sigma_{A d}^{\mathrm{H}}, \delta_{A d}\right\rangle$ such that

$$
\begin{aligned}
& -\Sigma_{A d}^{\mathrm{I}}=\left\{a \mid a \in \operatorname{Mismatch}_{\Phi}\left(A_{1}, A_{2}\right) \cap\left(\Sigma_{A_{1}}^{\mathrm{O}} \cup \Sigma_{A_{2}}^{\mathrm{O}}\right)\right\} ; \\
& \quad \text { For all a } \in \Sigma_{A d}^{\mathrm{I}}, \Psi_{A d}(a)=\Psi_{A_{1}}(a) \text { if a } \in \Sigma_{A_{1}}^{\mathrm{O}} \text {. Otherwise, } \Psi_{A d}(a)= \\
& \Psi_{A_{2}}(a) ; \\
& -\Sigma_{A d}^{\mathrm{O}}=\left\{a \mid a \in \operatorname{Mismatch}_{\Phi}\left(A_{1}, A_{2}\right) \cap\left(\Sigma_{A_{1}}^{\mathrm{I}} \cup \Sigma_{A_{2}}^{\mathrm{I}}\right)\right\} ; \\
& \quad \text { For all a } \in \Sigma_{A d}^{\mathrm{O}}, \Psi_{A d}(a)=\Psi_{A_{1}}(a) \text { if a } \in \Sigma_{A_{1}}^{\mathrm{I}} \text {. Otherwise, } \Psi_{A d}(a)= \\
& \quad \Psi_{A_{2}}(a) ; \\
& -\Sigma_{A d}^{\mathrm{H}} \subseteq\{\epsilon\} ; \text { in the adaptor this set represents the internal actions that do } \\
& \text { nothing, which are associated to input/output actions in mismatched compo- } \\
& \text { nents which are not concerned with the mapping (the adaptation); } \\
& -\delta_{A d} \subseteq S_{A d} \times \Sigma_{A d}^{\mathrm{I}} \cup \Sigma_{A d}^{\mathrm{O}} \cup\{\epsilon\} \times S_{A d} ; \\
& - \text { Shared }\left(A d, A_{1}\right)=\bigcup_{\alpha \in \Phi\left(A_{1}, A_{2}\right)} \Pi_{1}(\alpha) ; \operatorname{Shared}\left(A d, A_{2}\right)=\bigcup_{\alpha \in \Phi\left(A_{1}, A_{2}\right)} \Pi_{2}(\alpha) ;
\end{aligned}
$$

The adaptor must satisfy the following condition in order to ensure that the mapping rules are respected, therefore the mismatch between components is resolved.

The condition on the adaptor paths: For all execution path $\sigma=s_{1} a_{1} s_{2} a_{2} \ldots$ $s_{i} a_{i} \ldots s_{n}$ in the adaptor $A d$, such that $s_{i} \in S_{A d}$ and $a_{i} \in \Sigma_{A d}^{\mathrm{O}} \cup \Sigma_{A d}^{\mathrm{I}}$, if $\exists \alpha \in \Phi\left(A_{1}, A_{2}\right)$ such that the output actions (enabled as input in $A d$ ) of $\alpha$ are present in $\sigma$ then they are succeed, in $\sigma$, by there correspondent input actions (enabled as output in $A d$ ).

Property 1 An adaptor Ad for two interface automata $A_{1}$ and $A_{2}$ according to a mapping $\Phi\left(A_{1}, A_{2}\right)$ is composable with $A_{1}$ and $A_{2}$.

The property can be easily verified according to Definition 8 . Indeed, by considering the set of actions of $A d, \Sigma_{A d}^{\mathrm{I}}$ and $\Sigma_{A d}^{\mathrm{O}}$, the condition of composability, as defined in Section 4, can be easily verified with the set of actions of $A_{1}$ and $A_{2}$. 
S. Chouali et al.

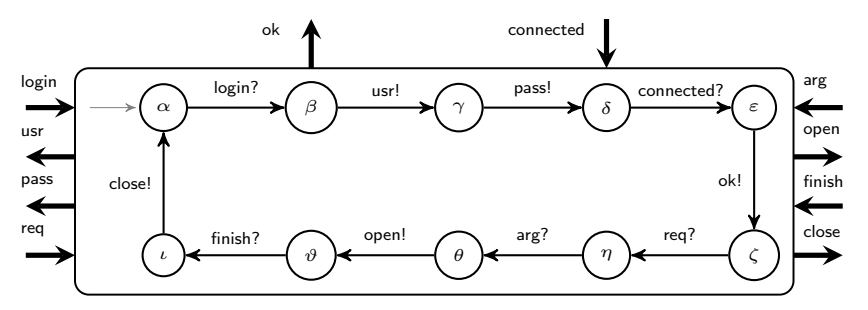

Fig. 2. The adaptor Adaptor for Client and Server

The composition of $A_{1}$ and $A_{2}$ is performed by synchronizing first $A d$ with either $A_{1}$ or $A_{2}$, computing their composition according to our extended approach, and then by composing the resulting composition with the remaining automaton. We suppose that the actions of the adaptor have the same signatures and semantics as actions in $\operatorname{Mismatch}_{\Phi}\left(A_{1}, A_{2}\right)$. If the composite interface automaton $A_{1}\|A d\| A_{2}$ is non empty then $A_{1}$ and $A_{2}$ are compatible after their adaptation at the protocol and the semantic levels.

To generate the adaptor $A d$ from $A_{1}, A_{2}$, and the mapping $\Phi\left(A_{1}, A_{2}\right)$, we have to explore in parallel the states and the transitions of both automata $A_{1}$ and $A_{2}$. For the lack of space, the details of the algorithm to perform adaptor generation is not described in this paper, however this algorithm is the same as our algorithm in [6] that constructs an adaptor for two composable interface automata $A_{1}, A_{2}$, and a given non empty mapping $\Phi\left(A_{1}, A_{2}\right)$. In fact the contribution of this paper compared to the approach in [6] is the handling of action semantics in component adaptability thanks to the design of components with behavioral contracts. The step for generating the interface automaton of the adaptor comes after verifying the semantic compatibility between $A_{1}$ and $A_{2}$. However in [6] we considered only the protocol level in the adaptation. So, the algorithm is basically a loop which reads in parallel $A_{1}$ and $A_{2}$ and constructs as one goes along the set of states and the set of transitions of the adaptor. The algorithm is executed by respecting the reordering of events of both interfaces $A_{1}$ and $A_{2}$. The algorithm marks and removes from the generated graph all the fragments of paths that do not respect the condition on the adaptor paths.

The part of the algorithm that constructs the set of states and transitions has the time complexity $\mathbf{O}\left(\left|S_{A_{1}} \times S_{A_{2}}\right| \cdot\left(\left|\delta_{A_{1}}\right|+\left|\delta_{A_{2}}\right|\right)\right)$. The time complexity of the part that removes the undesired path fragments is linear in the number of the generated states.

Example 4. As the reader can conclude, Adaptor is composable with both Client and Server presented in Example 1 and it satisfies all the items of Definition 8. Our proposed algorithm in [6] generates exactly the same interface automaton shown in Figure 2. Suppose that the semantic compatibility between the shared actions error and terminate holds, then Adaptor is compatible with both Client and Server. The composite interface automaton (Client $\|$ Adaptor) $\|$ Server is non empty which makes Client and Server compatible after their adaptation. 
Component Design and Adaptation Based on Behavioral Contracts

\section{Related Work}

Several techniques of adaptation show how to automatically derive adaptors in order to eliminate mismatches between components during their interactions. In [13], the authors propose an interesting approach based on finite state machines to adapt components specified by interfaces describing component protocol and action signatures. This approach deals with one-to-one relations between actions. In [8], the authors propose the Smart Connectors approach which allows the construction of adaptors using the provided and required interfaces of the components in order to resolve partial matching problems in COTS component acquisition. In [2], the authors have proposed a formal approach based on calculus to generate automatically adaptors using the Prolog language. The authors in [3] present an approach based on session types, exploited to specify component behaviors, to adapt heterogeneous components that may present mismatching interaction behaviors. In [7], Hemer has proposed, using template from the CARE language, to define adaptation strategies for modifying and combing components. In [9], the authors have proposed a model of adaptors expressed in the B formal method, allowing to define the interoperability between components. In [11] the authors introduce the concept of parameterized contracts and a model for component interfaces, they also present algorithms and tools for specifying and analyzing component interfaces in order to check interoperability and to generate adapted component interfaces. In [12], the authors propose to generate semi-automatically adaptors, at the protocol level, for concurrent components that are specified with finite state machines. Another approach that deals with the adaptation of component at the protocol level is presented in [10]. The authors proposed an algorithmic approach for checking whether incompatible interaction protocols of component interfaces can be made compatible by inserting a protocol converter between them. The approaches described above propose solutions for the component adaptation based on different specification formalisms of component interfaces. Our approach is different from the others, because we propose a solution to adapt particular components that are specified by interface automata. This formalism allows to exploit optimistic approach [1] to check to component interoperability. This adaptation approach deals with the signature, the semantic, and the protocol levels, and deals also with possibly complex adaptation scenarios : one-to-one and one-to-many correspondences between actions.

\section{Conclusion}

In this paper, we proposed a formal approach for the automatic development of component adaptors, allowing the elimination of mismatches between interacting components. Our component interfaces are described with behavioral contracts, which allow to handle all component facets for their adaptation, by considering component informations at levels of action signatures, their semantics, and their protocols. So, we proposed a formal framework for component adaptation, based on the following concepts: behavioral contracts, their composability, 


\section{S. Chouali et al.}

their synchronization, and their semantic compatibility. Therefore we specified these contracts with interface automata enriched by the action semantics. We exploited the obtained formalism to adapt the interface automata approach to verify compatibility between components specified with behavioral contracts. When components are incompatible due to mismatches, we proposed to specify a correspondence mapping between the mismatched actions of two components as a first abstract specification of the adaptor. This mapping deals with one-toone and one-to-many correspondences between the actions. Finally, we proposed an approach that generates the adaptor for two composable interface automata according to a fixed mapping. The generated adaptor allows to eliminate mismatches at signature, semantic, and protocol levels.

\section{References}

1. Alfaro, L., Henzinger, T.A.: Interface automata. ACM Press, 9th Annual Symposium of FSE (Foundations of Software Engineering) pp. 109-120 (2001)

2. Bracciali, A., Brogi, A., Canal, C.: A formal approach to component adaptation. Journal of Systems and Software 74, 45-54 (2005)

3. Brogi, A., Canal, C., Pimentel, E.: Behavioural types and component adaptation. In: Algebraic Methodology and Software Technology: 10th International Conference, AMAST 2004, volume 3116 / 2004 of LNCS. pp. 42-56. Springer-Verlag $\mathrm{GmbH}(2004)$

4. Canal, C., Murillo, J., Poizat, P.: Software adaptation. Special Issue on Software adaptation 12(1), 9-31 (2006)

5. Canal, C., Poizat, P., Salaün, G.: Synchronizing behavioural mismatch in software composition. Proc. of FMOODS'06, LNCS 6, 63-77 (2006)

6. Chouali, S., Mouelhi, S., Mountassir, H.: Adapting component behaviours using interface automata. IEEE Computer Society proceedings, Euromicro SEAA 2010 conference (September 2010)

7. Hemer, D.: A formal approach to component adaptation and composition. In Proceedings of the Twenty-eighth Australasian conference on Computer Science ACSC '05 Newcastle, Australia pp. 259-266 (2005)

8. Min, H., Choi, S., Kim, S.: Using smart connectors to resolve partial matching problems in cots component acquisition. LNCS, Springer-Verlag, Berlin, Germany 3054, 40-47 (2004)

9. Mouakher, I., Lanoix, A., Souquières, J.: Component Adaptation: Specification and Verification. In: 11th International Workshop on Component Oriented Programming (WCOP 2006). p. 8. ECOOP 2006, Nantes, France (07 2006)

10. Passerone, R., de Alfaro, L., Henzinger, T.A., Sangiovanni-Vincentelli, A.L.: Convertibility verification and converter synthesis: two faces of the same coin [ip block interfaces]. In: IEEE/ACM ICCAD 2002. pp. 132-139 (Nov 2002). https://doi.org/10.1109/ICCAD.2002.1167525

11. Reussner, R.: Automatic component protocol adaptation with the coconut/j tool suite. Future Generation Computer Systems 19(5), 627-639 (2003)

12. Schmidt, H., Reussner, R.: Generating adaptors for concurrent component protocol synchronisation. In the proceeding of the Fifth IFIP International Conference on Formal Methods for Open Object-Based Distributed Systems pp. 213-229 (2002)

13. Yellin, D., Strom, R.: Protocol specifications and components adaptors. ACM Transactions on Programming Languages and Systems 19(2), 292-333 (1997) 